\title{
ABSTRACTS OF CARDIOLOGY
}

\author{
EDITED BY J. L. LOVIBOND
}

Sequele of Deep Thrombosis in the Lower Limbs. Follow-up Study of Patients Initially Treated by Conservative Measures. I. C. HöJEnsGÅrD. Angiology, 3, 42-47, Feb., 1952.

At the University Hospital, Copenhagen, the author examined 109 patients who had suffered thrombophlebitis in the legs as a complication of parturition 2 to 31 years previously. None had been treated with anticoagulants: all had been treated by strict immobilization of the legs. Statistical analysis of the findings shows that in $89 \%$ of the patients symptoms or signs attributable to thrombosis were still present, thus confirming the view now held that the condition should be treated by means other than immobilization.

Cecil Flemming

Diseases of the Superior Vena Caval System, with Special Consideration of Pathology and Diagnosis. J. R. VeAL and N. J. Cotsonas. Surgery, 31, 1-12, Jan., 1952.

In this paper are discussed 137 patients with diseases of the great veins of the superior vena caval system. Of these patients 58 had a thrombosis of the axillary or subclavian veins, 50 an obstruction of the superior vena cava, and 11 an obstructed innominate vein. The remaining 18 had septic thrombophlebitis; in 14 of these, who were drug addicts, the cause was an infected injection of heroin.

C. G. Rob

Elastic Stockings in the Prevention of Pulmonary Embolism: a Preliminary Report. R. W. WILkINS, G. Mixter, J. R. Stanton, and J. LitTer. New Engl. J. Med., 246, 360-364, March 6, 1952.

The authors quote previous observations that an external pressure of 10 to $15 \mathrm{~mm}$. $\mathrm{Hg}$ applied to the leg decreases the effective vascular pressure and accelerates the venous flow. They refer to the tendency for older persons when confined to bed to develop venous thrombosis in the lower limb. With these observations in mind they have studied the effect on the incidence of pulmonary embolism of the wearing of elastic stockings by hospital patients.

There were no deaths from pulmonary embolism in a series of 2346 patients who wore elastic stockings continuously during their stay in hospital, although nonfatal emboli occurred in 2 cases. In a control group of 2395 patients who did not wear stockings, 4 had fatal and 12 non-fatal emboli. By contrast, clinical evidence suggesting local thrombotic disease in the legs occurred more often in the group who wore stockings (33 patients) than in the control group (27 patients). Patients with severe congestive cardiac failure and/or arterial disease of the lower limbs were excluded from both groups.

The authors conclude that elastic stockings do no harm, provided they are not applied too tightly, and that the results warrant further investigation into this method of prevention of pulmonary embolism. J. F. Goodwin

\section{Observations on the Surgical Treatment of Mitral Stenosis} by Commissurotomy. T. J. E. O'NeILl, R. P. Glover, and C. P. Bailey. J. Amer. med. Ass., 147, 10321033, Nov. 10, 1951.

This is a short summary from Philadelphia of the experiences of the authors in the operative treatment of 214 patients suffering from mitral stenosis. From a technical point of view they are very much in favour of division of the commissures with the knife rather than by "finger fracture." [Their results, when compared with those of the latter technique, scarcely justify this dogmatism.]

They classify their patients into five groups, varying from "Asymptomatic" to "Irretrievable," and confined their operations to those who fell within the three middle groups. Their results are assessed as follows: excellent, 89 (41.6\%); improved, 70 (32.7\%); unimproved, 29 (13.0\%); dead (all causes), 26 (12.6\%).

\section{J. R. Belcher}

Clinical and Physiological Correlations in Patients with Mitral Stenosis-V. B. M. LewIS, R. Gorlin, H. E. J. Houssay, F. W. Haynes, and L. Dexter. Amer. Heart J., 43, 2-26, Jan., 1952.

The area of the mitral valve and the degree of pulmonary vascular disease have been calculated from physiological data in 30 patients with pure mitral stenosis without evidence of active rheumatic carditis. From a hydraulic point of view, a valve area of $1.0 \mathrm{~cm} .2, \frac{1}{4}$ to $\frac{1}{6}$ normal, appears to be a critical one, for at that level of stenosis the pressure head needed to maintain a normal cardiac output approaches the plasma osmotic pressure. Probably from the stimulus of an increased pulmonary "capillary" pressure, a variable degree of vascular obstruction develops in patients when the mitral valve area becomes reduced to about $1.0 \mathrm{~cm} .{ }^{2}$ or smaller. This results in a lower cardiac output, which may protect the capillary bed from sudden increases in pressure.

The clinical picture in mitral stenosis can be explained in a large measure by the interplay of the degree of stenosis with the degree of pulmonary vascular obstructions. The combination of a narrow valve with only a slight increase in pulmonary arteriolar resistance is associated with predominantly respiratory symptoms, exer- 
tional dyspnœa, hemoptysis, and paroxysmal nocturnal dyspncea, the right ventricle not becoming dilated. The effect of a narrow valve and a high pulmonary arteriolar resistance appears to be additive in causing electrocardiographic evidence of right ventricular hypertrophy and roentgenographic signs of cardiac enlargement. The combination of a narrow valve and great increases in pulmonary arteriolar resistance is associated with severe dyspnœa, cardiac enlargement, and signs of right ventricular failure.

Most patients with mitral stenosis can be divided into four general categories by their signs, symptoms, and physiological adjustments.-[Authors' summary.]

Failure of Ligation of the Left Auricular Appendage in the Prevention of Recurrent Embolism. F. C. LEONARD and M. A. Cogan. New Engl. J. Med., 246, 733-735, May 8, 1952.

The authors point out that once a patient has had an embolism in an attack of rheumatic heart disease he may have a recurrence of the embolism at any time. The majority of emboli are thought to originate from the left auricular appendix, and therefore this structure has been amputated in the hope of preventing a clot from forming and spreading. It would appear, however, that this procedure is not adequate as a clot may well form within the cavity of the auricle proper.

The administration of dicoumarol and of anticoagulants over a prolonged period has proved to be the best treatment so far, and the authors consider that unless clots within the heart can be localized resection of the auricular appendix should be abandoned.

\section{T. Holmes Sellors}

Chronic Constrictive Pericarditis-I. Some Clinical and Laboratory Observations. V. A. McKusick. Bull. Johns Hopk. Hosp., 90, 3-26, Jan., 1952.

Clinical details are given of 20 cases of constrictive pericarditis. The pressure curves obtained from the right ventricle were characterized by an early diastolic fall (" dip ") in pressure followed by a rapid rise to a plateau at a high pressure level. This rapid rise in pressure in early diastole is presumably related to the reduced distensibility of the right ventricle as blood flows in from the auricle. A dip also occurred in the auricular pressure curve taken at the same moment, and in one case at operation an identical type of pressure record was obtained from the left ventricle. The pressure dip is closely related to the protodiastolic sound occurring in constrictive pericarditis. Tuberculosis plays a part in ætiology in some cases, but in others trauma may be important. The serum albumin level is occasionally low in constrictive pericarditis, but this may recover very rapidly after operation. Radiologically, dilatation of the superior vena cava with little cardiac enlargement is always suggestive, but localized constriction at the caval orifices was not found. Calcification was present in only half the cases. The condition may closely simulate mitral stenosis.

The results of pericardectomy were, in general, satisfactory. The only deaths that occurred were in patients over 55 years of age.
The Responsibility of the Physician in the Selection of Patients with Mitral Stenosis for Surgical Treatment. D. W. Harken, L. B. Ellis, L. DeXter, R. E. FarRAND, and J. F. DiCkson. Circulation, 5, 349-362, March, 1952.

Examination of 35 normal hearts has shown that in addition to the ventricular and aortic leaflets there are two further small leaflets, the whole constituting a continuous veil round the circumference of the mitral orifice. In $85 \%$ of cases mitral stenosis is due to a rigid contraction of the leaflets without fusion of the chorda tendineæ. Calcification is common in this type. In the remaining $15 \%$ an elastic funnel results from fusion of the chordæ. If the mouth of the funnel is turned towards the ventricular wall, ventricular contraction may close it during systole; if, however, the opening faces into the ventricular outflow tract, free regurgitation may take place. Calcification is rare in this group.

Finger-fracture valvuloplasty is regarded as the operation of choice for the first group, and may seem to restore the valve to normal. But there is no overlap of the leaflets, so that the valve would not remain competent if the ventricular pressure was high, as in aortic stenosis or hypertension. In the second group a valvulotome, fitted along the finger, may be needed to incise some of the chordæ.

Patients with mitral stenosis are classified as follows: (1) those without symptoms, who do not require operations; (2) those with dyspncea on effort, who should be watched closely in case they pass into the next stage; (3) those with increasing dyspncea and signs of pulmonary congestion (operation is urgently required for these, since they may die from pulmonary cedema); and (4) those with right ventricular failure, who should have had a valvulotomy earlier. The hazards are greater in these last, but operation should still be considered, as sometimes dramatic improvement follows. Active rheumatic carditis and severe aortic diseases are regarded as absolute contraindications, and mitral regurgitation as a relative contraindication. Extensive valvular calcification, auricular fibrillation, or a history of previous emboli are all considered to increase the risk.

$$
\text { C. W. C. Bain }
$$

Treatment of Subacute Bacterial Endocarditis with Aureomycin. C. K. Friedberg. J. Amer. med. Ass., 148, 98-103, Jan. 12, 1952.

At the Mount Sinai Hospital, New York, 11 patients with subacute bacterial endocarditis were treated with aureomycin, 4 to $6 \mathrm{~g}$. daily being given 6-hourly by mouth. In 6 of the patients the causal organism was Streptococcus viridans and in 2 Strep. faecalis; no organism was isolated from 3 of the patients.

Aureomycin effected a cure in 2 of the cases due to Strep. viridans and in 2 of the 3 patients with negative blood cultures. Of the 6 patients with positive blood cultures who failed to respond to aureomycin 5 were subsequently cured with penicillin or a combination of antibiotics. In most of the cases administration of aureomycin was followed by clinical improvement, abolition of fever, and negative blood cultures, but the disease relapsed shortly after treatment was stopped. 
This study supports the view that the action of aureomycin is mainly bacteriostatic as opposed to the bactericidal action of penicillin. It is concluded that aureomycin should not be used as a routine for the treatment of subacute bacterial endocarditis, but that its use, alone or combined with other antibiotics, is indicated in cases failing to respond to penicillin or infected with organisms resistant to penicillin.

C. Bruce Perry

Myocardial Lesions in Subacute Bacterial Endocarditis.

E. L. Perry, R. G. Fleming, and J. E. Edwards. Ann. intern. Med., 36, 126-137, Jan., 1952.

The myocardium was studied in 43 untreated cases of subacute bacterial endocarditis and in 9 cases treated with penicillin at the Mayo Clinic. Miliary infarcts in varying stages of development were found in $90 \%$ of all cases, and non-specific interstitial inflammatory lesions and perivascular lesions were found in $50 \%$ of the cases. These were regarded as embolic. In the treated group there was a greater tendency for the lesions to heal and the production of more chronic reactions. There appeared to be poor correlation between the myocardial lesions and the incidence of cardiac failure.

\section{R. H. Heptinstall}

Use of Quinidine in Treatment of Chronic Auricular Fibrillation. Results Obtained in a Series of One Hundred and Fifty-five Patients. E. H. YounT, M. Rosenblum, and R. L. McMillan. Arch. intern. Med., 89, 63-69, Jan., 1952.

On the assumption that auricular fibrillation by itself may have deleterious effects on cardiac function, the authors have given oral quinidine-to which, they say, there are no contraindications except sensitivity-to 155 patients with chronic fibrillation, due in most cases to atheroma and rheumatic heart disease. In no fewer than 119 patients was sinus rhythm re-established in an average of 6 days with doses of $0.4 \mathrm{~g}$. 4-hourly and a mean plasma concentration of $9 \mathrm{mg}$. quinidine per litre. No relation was found between age, cardiac failure, or duration of fibrillation and inability to revert; and since reversion invariably took place at a blood level of 8 to $12 \mathrm{mg}$. per litre it was not considered justifiable to increase dosage indefinitely in patients who did not respond. A therapeutic blood level was attained in a few hours, with cumulation over the first 3 days; when the drug was discontinued the blood level fell rapidly, and it seems probable that quinidine is destroyed in the body. The authors consider that about half the therapeutic dose is required for maintenance and may be given 6-hourly.

Toxic symptoms-chiefly gastro-intestinal and vestibular-occurred at a therapeutic blood level and may have been due to the rapidity of rise. It is suggested that the development of flutter is no reason for stopping quinidine, and the previous occurrence of emboli is regarded as an urgent indication for the drug; no embolic episodes occurred during treatment. Only 41 patients have so far been traced, and 6 of these have relapsed in an average period of 6 months after reversion.

A. Paton
Complete Functional Recovery after Coronary Occlusion and Insufficiency. A. M. MASTER and H. L. JAFFE. J. Amer. med. Ass., 147, 1721-1726, Dec. 29, 1951.

During the past 25 years the authors were called into consultation in 554 cases of acute coronary occlusion. Of this total 79 patients, of whom 35 died, were seen only in the acute attack, and for various reasons a satisfactory follow-up was not possible in another 63; these patients were excluded from the present investigation. An additional 141 patients were investigated in whom a diagnosis of acute coronary insufficiency had been made. The distinction between coronary insufficiency and occlusion was made on electrocardiographic findings, coronary insufficiency being diagnosed when the $T$-wave changes and the RS-T depression appeared early and returned to normal in several days or weeks, whereas coronary occlusion was diagnosed when the changes are more marked and more prolonged. The patients were followed up for a period averaging $6 \frac{1}{2}$ years (and ranging from 3 months to 29 years).

Of the 412 patients with coronary occlusion, 154 $(37 \cdot 5 \%)$ made a good recovery, and $115(28 \%)$ made a satisfactory recovery but continued to have occasional effort angina.

Of the 154 patients who made a complete functional recovery, only $6(4 \%)$ survived less than 5 years, and the total mortality in this group (19\%) was less than half of that among the 115 patients who continued to have symptoms (47\%);25\% survived more than 10 years, and $40 \%$ more than 5 years, $83 \%$ being employed during that time. Those who were less than $\mathbf{4 5}$ years old at the time of the attack did only slightly better than the older patients. The best prognostic guide after coronary occlusion thus appears to be the patient's symptoms rather than the objective findings. Whether an infarct was anterior or posterior did not appear to influence the future course. The prognosis following acute coronary insufficiency was better than that after coronary occlusion. Only $4 \%$ of these patients died, and more than half of them made a complete recovery.

The results as a whole indicate that most patients who survive an attack of coronary occlusion or insufficiency may be encouraged to lead fairly normal lives.

\section{H. E. Holling}

"Armchair " Treatment of Acute Coronary Thrombosis. S. A. Levine and B. Lown. J. Amer. med. Ass., 148, 1365-1369, April 19, 1952.

In 81 cases of acute coronary thrombosis treated at home and at the Peter Bent Brigham Hospital, Boston, the patients were encouraged at the end of the first week of their illness to spend the greater part of each day sitting in a comfortable chair with their legs dependent; shock, marked debility, or cerebral vascular accidents were the only contraindications. The authors hold the view that this method reduces cardiac output, discourages pulmonary congestion, and reduces the incidence of thrombophlebitis and other complications. All the other methods of treatment usually employed in coronary thrombosis were also used.

In this uncontrolled series, in which there were 8 deaths $(9.9 \%)$, the results were held to be satisfactory, 
and the method appeared especially valuable in patients with congestive heart failure. The beneficial psychological effect of the manœuvre in reducing feelings of anxiety is also stressed. J.W. Litchfield

Indications for Bishydroxycoumarin (Dicoumarol) in Acute Myocardial Infarction. H. I. RUSSEK, B. L. ZOHMAN, A. A. Doerner, A. S. Russek, and L. G. White. Circulation, 5, 707-711, May, 1952.

A series of 623 patients suffering from myocardial infarction were divided into two groups according to the history and the facts elicited at a physical examination carried out during the first 24 hours in hospital. In the first group were 338 " poor risk" patients, in whom one or more of the following were noted: evidence of previous myocardial infarction; intractable pain; extreme degree or persistence of shock; marked cardiac enlargement; gallop rhythm; congestive heart failure; auricular fibrillation or flutter, ventricular tachycardia or intraventricular block; diabetic acidosis; marked obesity; varicose veins; or evidence of previous thromboembolism. In the second group were 285 patients in whom none of these signs or symptoms was noted and these were classified as "good risk" patients. All the patients were treated conservatively. In the first group the mortality in hospital was $70 \%$ and the incidence of thrombo-embolism was $12 \cdot 4 \%$, compared with $3 \cdot 5 \%$ and $0.7 \%$ respectively in the second group.

The authors suggest that it is pointless to subject the second group, which were mild cases, to the hazards of anticoagulant therapy for the sake [as they calculate] of a theoretically preventable mortality of about $1 \%$.

R. S. Stevens

Chronic Cardiac Aneurysm. (Das chronische Herzaneurysma). B. Steinman and M. Elsaesser. Helv. med. Acta, 19, 42-65, March, 1952.

This article is based on personal observation of 31 cases of aneurysm of the heart and includes a good review of the literature. The authors suggest that the condition is not so rare as is generally supposed and that its clinical, radiological, and electrographic signs should be more carefully looked for. A persistently raised S-T segment in the chest leads taken over the left ventricle (the most frequent site of aneurysm) is very suggestive of its presence.

G. S. Crockett

Observations on Some Possible Precursors of Essential Hypertension and Coronary Artery Disease. C. B. ThOMAs. Bull Johns Hopk. Hosp., 89, 419-441, Dec., 1951.

At the end of the first 4 years a preliminary report is submitted on some 400 medical students of Johns Hopkins University who have been taking part in an investigation designed to elucidate the importance of some traits most frequently associated with the early onset of hypertension and coronary artery disease. This investigation included detailed heredity studies, studies of the cardiovascular system at rest and under stress, metabolic investigations, and personality studies. In no fewer than 93 out of 353 students there was a history of hypertension or coronary artery disease in at least one parent. In this group with a " positive" parental history there appeared to be a significant proportion of subjects with high resting blood pressure and heart rate, transitory tachycardia, and hyperreactivity to the cold pressor test and to exercise tests; $36 \%$ of 332 subjects showed evidence of hypercholesterolæmia.

[The hereditary factor in essential hypertension and coronary artery disease is now undisputed. It remains to be seen what, if any, significance can be attached to the finding of other "precursors" of hypertension and coronary atherosclerosis; the follow-up results in these (now apparently healthy) young subjects, extending over many decades, should be of some help.]

\section{A. I. Suchett-Kaye}

The Size of the Heart during the Course of Essential Hypertension. M. KLeINFEld and J. ReDish. Circulation, 5, 74-80, Jan., 1952.

The transverse diameter of the heart of 45 hypertensive patients was observed by teleradiography over a period of 5 to 20 years. It was found that 10 patients had normal measurement for 5 to 10 years, and 13 for more than 10 years. The degree of enlargement was assessed by comparing actual size with the predicted size as given in the tables of Ungerleider and Clark, and in some cases by fluoroscopy.

Progressive enlargement was seen in 24 patients, while in 18 the heart size remained stationary; 3 patients showed sudden increase in size followed by progressive enlargement. Cardiac failure might occur in patients with normal heart measurements, and marked enlargement might be present for 10 or more years with little functional impairment. Cardiac infarction might lead to sudden enlargement or be followed by no change in heart size. Reduction of body weight by 20 to $40 \mathrm{lb}$. (9 to $18 \mathrm{~kg}$.) did not reduce the size of the heart.

The authors conclude that the size of the heart has, by itself, little value in deciding the prognosis in essential hypertension. J. W. Litchfield

The Treatment of Hypertension with the Kempner Diet and a Low-sodium Regimen. Clinical Observations on 600 Cases. F. Montoreano and J. Rabenko. Prensa méd. argent., 38, 2849-2856, Nov. 2, 1951.

This is an account from Buenos Aires of a study of 600 patients between the ages of 28 and 79 with essential hypertension, who were treated with Kempner's rice diet. The diet was given for periods varying from 2 weeks to 4 months; some patients took a modified Kempner's diet intermittently up to 2 years. The majority of patients refused the diet after a time. In about $90 \%$ improvement of symptoms was noted, but $60 \%$ complained of weakness. As regards the effect on blood pressure, the systolic pressure was reduced to normal in $69(11.5 \%)$; it fell by over $50 \mathrm{~mm}$. $\mathrm{Hg}$ in $156(26 \%)$, $30 \mathrm{~mm}$. $\mathrm{Hg}$ in $177(29.5 \%)$, and up to $20 \mathrm{~mm}$. $\mathrm{Hg}$ in $174(29 \%)$; no change was noted in $57(8 \%)$; and there was a rise in $36(6 \%)$. The diastolic pressure was reduced to normal in $135(22.5 \%)$; it fell by over $20 \mathrm{~mm}$. $\mathrm{Hg}$ in $87(14.5 \%), 15 \mathrm{~mm}$. $\mathrm{Hg}$ in $174(29 \%)$, and up to $10 \mathrm{~mm}$. $\mathrm{Hg}$ in $168(28 \%)$; there was no change in $144(24 \%)$, and a rise in $27(4 \%)$. The heart 
size was determined radiologically in 106 cases, a reduction being observed in 35 . The main change in the electrocardiogram consisted in a reduction in S-T depression. After the rice diet was stopped the blood pressure started to rise in about a fortnight, regaining its original level after a period varying between a few days and several months.

It is concluded that this method is effective in reducing the blood pressure without, however, modifying the course of the disease.

A. Schoit

\section{Hypertensive Cardiovascular Disease. Effect of Thoraco- lumbar Splanchnicectomy on Mortality and Survival Rates. R. H. Smithwick. J. Amer. med. Ass., 147, 1611-1615, Dec. 22, 1951.}

The author has attempted to determine the effect of standard lumbo-dorsal sympathectomy on the prognosis in hypertensive cardiovascular disease by comparing the mortality and survival rates in a series of cases treated surgically at the Massachusetts Memorial Hospital, Boston, with those in comparable cases treated medically at the same hospital or elsewhere. In classifying cases according to the severity of the disease, two methods of assessment are used, that of Keith, Wagener, and Barker (Amer. J. med. Sci., 1939, 197, 332) and that devised by the author. The former authors analysed a series of 219 cases of hypertension treated non-surgically, dividing them into four groups according to the severity of the changes in the optic fundus, to which they showed the prognosis to be closely related. These patients were followed up for a minimum of 5 years, and of the present author's series, 192 of the 296 patients treated medically had been observed for a similar period. The mortality rates among these 192 patients, when classified on the same basis, were found to bear a marked similarity to those in the earlier series, and the two series are therefore combined to provide a total of 411 medically treated cases, as against 538 surgically treated cases with a comparable follow-up period. The mortality rates in the latter show a very impressive reduction on those in the former in all four groups at 5 years, the difference being maintained to the end of the 5- to 10-year follow-up period. The most striking feature is the survival of $44 \%$ of surgically treated patients in Group 4 at the end of the period, compared with only $0.5 \%$ of those treated medically.

The author then classifies his whole series of 892 cases (596 treated surgically and 296 medically), in which the follow-up period ranged from 4 to 12 years, into four groups according to his own system, which takes account of various manifestations of hypertensive disease in addition to fundus changes. Again the comparison of mortality rates is greatly in favour of operation in all groups at 4 years and at the end of the follow-up period, the difference being most marked in the author's Groups
2 and 3. These groups include patients with any grade of fundus change and with cerebral, cardiac, and/or renal changes whose diastolic pressure is not above $140 \mathrm{~mm}$. $\mathrm{Hg}$ and who are not suffering from severe renal damage, congestive heart failure, or the residuum of a cerebrovascular accident: the author considers that surgery is of real value in these two groups. For patients in his Group 1 (uncomplicated diastolic hypertension with a good prognosis), he concludes that surgery cannot as yet be justified on a statistical basis, whereas patients in Group 4 -those with the most severe form of the disease -may benefit to a worth-while extent in the absence of nitrogen retention, which is considered an absolute contraindication to operation. Survival curves for the surgically and non-surgically treated cases in each group are given.

C. J. Longland

Hypertension and the Surgical Kidney. A. D. PUPPEL and E. P. AlYeA. J. Urol., 67, 433-440, April, 1952. The authors have studied the incidence and behaviour of hypertension coexisting with renal lesions in patients coming to nephrectomy and compared their findings with those in hypertensive patients without renal lesions.

In 23 of 67 patients with hypertension at the time of nephrectomy, which was performed for other reasons, blood pressure was normal two or more years after operation. This was most commonly seen after removal of a hydronephrotic kidney. The incidence of surgical renal lesions in hypertensive patients is only $2 \%$ and routine pyelograms are not, therefore, considered to be justified in hypertension. The incidence of hypertension is not significantly higher in patients admitted to hospital for surgical renal disease than in those admitted for other reasons.

J. Marshall Pullan

Clinical Use of Cation Exchange Resins in the Treatment of Congestive Heart Failure. E. J. Callahan, N. R. Frank, H. Kraus, and L. B. Ellis. Amer. J. med. Sci., 223, 117-125, Feb., 1952.

The authors reaffirm the value of a cation-exchange resin (an ammonium-potassium cycle carboxylic resin) in the treatment of congestive cardiac failure. Patients receiving the resin were able to eat a more liberal diet, particularly as regards sodium, which was made unabsorbable by the resin. Mercurial diuretics could be reduced while the patients were taking the resin.

All toxic effects were manifested clinically, and the authors consider that elaborate laboratory studies are not necessary in following the progress of the patients. Gastro-intestinal symptoms, such as nausea, anorexia, vomiting, and constipation, were the commonest complications of treatment. Digitalis intoxication also occurred fairly often, but the reason for this remains obscure. The commonest electrolyte disturbances were those of hypocalcæmia and hypokalæmia, with a compensated acidosis.

IV Congrès National de Cardiologie will be held at Séville, Spain on May 7-10, 1953. Any member of the British Cardiac Society wishing to attend should communicate with the Secretary of the British Cardiac Society. 